\title{
Bad Breath
}

\section{Sagunthala Ettikan \\ Iv Bds Undergraduate}

\begin{abstract}
Bad breath is related to poor oral hygiene is most common and caused by release of sulphur compounds by bacteria in the mouth.There are several causes of bad breath such as diet,drymouth,tobaccoproducts,medicaldisorders,denture or dental appliances and morning breath.Organoleptic measurements rank the intensity of odours which are considered the criterion standard for the measurement of malodour.Bad breath can be analysed by instrumental analysis and bacteriological analysis.Instrumental analysis which level of intraoral volatile sulphur compounds (VSCs) can be estimated while bacteriological analysis is a complete head and neck examination including nasal endoscopy,flexible laryngoscopy and sites directed cultures are indicated.This oral malodour can be treated by maintaining good oral hygiene practices such as flossing, care of denture,mouthwash and diet.
\end{abstract}

\section{Introduction}

Bad breath is breath that has an unpleasant odour. It's also known as halitosis. Thisodour can occur from time to time or it can be long lasting which is depending on cause.[1]

Malodour that arises from the mouth is the consequence of microbial putrefaction of food debris, cells, saliva and blood. The role of volatile sulfur compounds (VSCs) in producing bacteria that colonize over the tongue was recently understood as a main cause of halitosis. The common VSCs such as methylmercaptan and hydrogen sulphite can be detected by using organoleptic. The oral microbes most likely to cause the oral malodour are Gram negative bacteria and include Prevotellamelaninogenica ,Treponemadenticola,Propyromonasgingivalis,Propyromonasendodontalis,Enterobacteriacae and Fusobacteriumnucleatumvicentii.[4]

You may not always know that you have bad breath. That's because odour detecting cells in the nose eventually get used to the smell, other people may notice and react by stepping away from you as you speak or making a face. Some common symptoms of bad breath include bad breath smell, bad taste or taste changes, dry mouth and a coating on the tongue. Besides that symptoms such as infection in the mouth, respiratory tract infection, dry mouth and illness cause bad breath.[1]

There are many things that can cause bad breath including diet. When a person does not brush teeth thoroughly, food particles may remain in the mouth these particles may rot and cause foul odours. Bad breath can also caused by decreased flow of saliva, which is vital part of the digestive and removes odour causing particles in the mouth also called xerostomia.in addition to causing bad breath smoking or chewing tobacco based products can stainteeth, irritate gum tissue and exacerbate tooth decay. Certain medical disorders can cause bad breath, for example gum or periodontal infection,diabetes, GERD and denture or dental appliances can contribute to bad breath.[5]

Oral malodour can be measured studies using organoleptic intensity and organoleptic hedonic indices and instruments that quantitate the amount of volatile compounds or bacterial enzymes that contribute to the production of odiferous compounds. [2]

People who suffer from bad breath want to know to get rid of bad breath. Some measures an individual can do to prevent or get rid of bad breath include practice good oral hygiene, stopsmoking, keep mouth moist ,avoid food such as anions or garlic, mouthwash and natural bad breath remedies include chewing on mint or parsley.[3]

In nut shell, oral malodour is primarily result of microbial metabolism. Oral malodour from the overgrowth of proteolytic, anaerobic Gram negative bacteria on the crevices of the dorsum can be successfully diagnosed and treated.

\section{Definition}

Bad breath is breath that has unpleasant odour. The medical term for it is halitosis, Fetorosis, or Stomatodysodia. Halitosis is common among humans and is usually caused by an accumulation of bacteria in the mouth as a result of gum disease, food or plaque. [7] 
III. Classification:

\begin{tabular}{|c|c|c|}
\hline Classification & Treatment & Description \\
\hline I. Genuine Halitosis & & $\begin{array}{l}\text { Obvious malodor, with intensity beyond } \\
\text { socially acceptable level, is perceived. }\end{array}$ \\
\hline A.Physiologic Halitosis & TN-1 & $\begin{array}{l}\text { 1. Malodor through putrefactive process } \\
\text { within the oral cavity. Neither specific } \\
\text { disease nor pathologic disease could cause } \\
\text { halitosis is found. } \\
\text { 2. Origin is mainly the dorsoposterior region } \\
\text { of tongue. } \\
\text { 3. Temporary halitosis is due to the dietary } \\
\text { factor (e.g.: garlic) should be excluded. }\end{array}$ \\
\hline \multicolumn{3}{|l|}{ B. Pathologic halitosis } \\
\hline i)Oral & $\mathrm{TN}-1$ and $\mathrm{TN}-2$ & $\begin{array}{l}\text { 1. Halitosis caused by disease, pathologic } \\
\text { condition or malfunction of oral tissues. } \\
\text { 2. Halitosis derived from tongue coating } \\
\text { modified by pathologic condition (e.g.: } \\
\text { periodontal disease, xerostomia) is included } \\
\text { in this subdivision. }\end{array}$ \\
\hline ii) Extra-oral & TN-1 and TN-3 & $\begin{array}{l}\text { 1. Halitosis originates from nasal, paranasal } \\
\text { and laryngeal regions. } \\
\text { 2. Malodor originates from disorders } \\
\text { anywhere in the body whereby the odor is } \\
\text { blood borne and emitted via the lungs. (e.g.: } \\
\text { diabetes mellitus, internal bleeding) }\end{array}$ \\
\hline II. Pseudo-Halitosis & TN-1 ad TN-4 & $\begin{array}{l}\text { 1. Obvious malodor is not perceived by } \\
\text { others although patient stubbornly } \\
\text { complains about the existence. } \\
\text { 2. Condition is improved by counseling } \\
\text { (using literature support, education and } \\
\text { explanation of examination results) and } \\
\text { simple oral hygiene measures. }\end{array}$ \\
\hline III. Halito-Phobia & TN-1 and TN-5 & $\begin{array}{l}\text { 1. After treatment for genuine halitosis or } \\
\text { pseudo-halitosis, the patient persists in } \\
\text { believing that they have halitosis. } \\
\text { 2. No physical or social evidence exists to } \\
\text { suggest that halitosis is present. }\end{array}$ \\
\hline
\end{tabular}

\section{Ken Yaegakietal2000 [8]}

\section{Source:}

The mouth is home to hundreds of bacterial species with various nutritional preferences. Malodour that arises from the mouth is the consequence of microbial putrefaction of food debris, saliva and blood. [4]

The role of VSCs in producing bacteria that colourize over the tongue is causing halitosis. The most common VSCs such as methylmercaptan, and hydrogen sulfite. [2]

The oral microbes most likely to cause oral malodour are Gram negative bacteria and include Prevatella melaninogenia, Treponema denticola, Porphyromonas gingivolis, Fusobacterium nucleatum and etc. [4]

\section{Symptoms:}

You may not always know that you have a bad breath. That's because odour detecting cells in nose eventually get used to the smell. Other people may notice and react by stepping away from you as you speak.

Other Symptoms:

-Infection in the mouth:

Red or swollen gums that may bleed easily, especially after brushing or flossing.Pus between teeth or a pocket of pus(abscess) at the base of tooth.Loose teeth or a change in how the denture fits. Painful, open sores on the tongue or gums.:[5]

-Respiratory Tract infection:

Respiratory infections such as sore throat,swollen lymph nodes in the neck,fever,stuffynose.a greenish or yellowish discharge from the nose and a cough with mucus. :[5]

-Dry Mouth

Difficulty swallowing food.Difficulty in speaking for a long time due to mouth dryness.Burning in the mouth.An unusually high number of cavities and dry eyes.:[5] 
-Illness

Ilness such as diabetes,lungdisease,kidney failure and liver disease:[5]

\section{Duration:}

The duration of bad breath is dependent on its cause. For example, if it is caused by poor dental hygiene, proper dental care begins to freshen the mouth right away. You will have even better results after a few days of regular brushing and flossing. Periodontal diseases and tooth abscess also respond quickly to proper dental treatments. Bad breath caused by chronic sinusitis may keep coming back especially if it is caused by structural abnormality of the sinuses. Bad breath that results from an illness may be a long term problem. It often can be controlled with proper medical care. $[1,5]$

\section{Pathogenesis:}

In people with rigorous and hygienic good dentition and a healthy periodontium, the main cause of bad breath is likely to be the back of the tongue. People whose tongues are deeply grooved or furrowed have more potential to accumulate the white coating than those without. A coating of $0.1-0.2 \mathrm{~mm}$ thick can deplete an environment of oxygen. The bacteria that cause bad breath can flourish in this type of anaerobic environment.

Dentures are another important cause of oral malodour, particularly if they are worn overnight. Usually the odour has a somewhat sweetish but unpleasant typical character and is really identifiable particularly if the dentures are placed in a plastic bag and smelled after several minutes.

Saliva plays a big role in bad breath elimination. Bad breath levels during the day are inversely related to saliva flow. When saliva is lowest at night, following fasting and due to insufficient water intake, the intensity of bad breath rises. Conversely, mastication increases saliva flow with contaminant cleaning of the oral cavity and reduction in malodour. One possible explanation for the latter observation is that malodor arises primarily in an alkaline microenvironment, whereas the saliva in patients with xerostomia is often acidic. The most common bacterium was Streptococcus salivarius. This bacterium was present only in 1 out of 6 people with halitosis, at extremely low levels.

The role of tonsils in chronic bad breath is unclear. The appearance of a transient odour during tonsil infections in adults and children is common. Though they may appear normal upon visual examination, when tonsils are pressed with a tongue blade, they can emit foul smelling exudates. Dilated and deep tonsillar crypts may contain tonsillitis. They are usually several millimetres in diameter, rough edged, white or yellowish in color, particularly when pressed :[5]

\section{Causes:}

-Diet And Poor Oral Hygiene

Diet and poor oral hygiene when a person does not brush or floss their teeth thoroughly, food particles remain in the mouth. These particles may rot and cause foul odours. In addition, food particles over time can promote the bacterial growth which also causes bad odour. The bacteria can also lead to tooth decay and gum disease.

Food with strong odours also affects the air a person exhales. Food commonly known to contribute to bad breath include onions and garlic, exotic spices, cheese, fish and acidic beverages such as coffee. Food are absorbed into bloodstream and then transferred to the lungs, causing noticeable odours when exhaled. This food may also cause gastrointestinal upset which can contribute to bad breath. In addition, certain supplements such as fish oil capsules can contribute to bad breath.

Low carbohydrate diets may also cause 'ketone breath', so called 'low carbs' diets cause the body to burn fats as its energy source. The end product of making this energy is ketones, which cause a fruity acetone like odour on the breath when exhaled. :[5]

\section{•DRY MOUTH}

Bad breath can also be caused by decreased flow of saliva, which is vital part of the digestive process and removes odour causing particles in the mouth. Also called xerostomia, dry mouth may be caused by medications, breathing through the mouth or salivary gland problems. :[5]

\section{-TOBACCO PRODUCTS}

It does not only cause bad breath but also stains the teeth, irritate gums and causes tooth decay when tobacco is smoked or chewing of the tobacco based products.:[5]

\section{-MEDICAL DISORDERS}

Medical disorders such as Gum or periodontal infection,throatinfection (pharyngitis or tonsillitis),local infection in respiratory tract and chronic sinusitis or post nasal drop. Besides that,diabetes,gastroesopharyngeal 
reflux disease (GERD),liver or kidney disease,Sjogren's syndrome and lactose intolerance which leads to bad breath.:[5]

\section{-DENTURE OR DENTAL APPLIANCES}

Dental appliances such as braces can contribute to bad breath. Most often is due to food particles that are not properly cleaned from the appliances. Loose fitting dentures can contribute to sores and localized infections in the mouth which can cause bad breath. :[5]

\section{•MORNING BREATH}

Overnight, bacteria accumulate in the mouth, causing bad breath that is commonly referred to as 'morning breath'. Some people breathe through their mouth at night, which can cause dry mouth and worsen the morning breath. [5]

\section{Diagnosis:}

Organoleptic measurements rank the intensity of odors are considered the criterion standard for the measurement of malodor. Either a 5-point or a 10-point scale can be used and usually 2 separate judges evaluate the degree of halitosis. :[ 2] The intensity is based on Rosenberg scale, which rates odor intensity and is as follows:

Organoleptic Scoring Scale

\begin{tabular}{|l|l|}
\hline Category & Description \\
\hline 0: Absence of odor & Odor cannot be detected. \\
\hline 1: Questionable odor & $\begin{array}{l}\text { Odor is detachable although the examiner couldn't recognize it as } \\
\text { malodor. }\end{array}$ \\
\hline 2: Slight malodor & Odor is deemed to exceed the threshold of malodor recognition. \\
\hline 3: Moderate malodor & Malodor is definitely detectable. \\
\hline 4: Strong malodor & Strong malodor is detected, but can be tolerated by examiner. \\
\hline 5: Severe malodor & $\begin{array}{l}\text { Overwhelming malodor is detected and cannot be tolerated by the } \\
\text { examiner. }\end{array}$ \\
\hline
\end{tabular}

\section{Ken Yaegakietal 2000 [8]ANALYSIS:}

a) Instrumental analysis

The level of intraoral volatile sulfur compounds (VSCs) can be estimated chair side, using portable sulfide monitors such as Halimeter. Sensors for VSCs have been incorporated into probes and paddles, which can be placed directly on the tongue for measurement. [Diamond Probe, Ann Arbor, Michigan]The recent advances are in the field of a VSC monitor which uses a zinc oxide sensor for an objective quantification of halitosis. Advantage of the tests mentioned above, clinician interested in diagnosing bad breath must still rely on their noses to distinguish the main type of oral odours. These include periodontal type odour which usually comes from periodontal pockets and interdental spares,odour from the posterior tongue dorsum,denture odour and characteristic nasal odour[2]

b)Bacteriological Analysis

Given the multiple sites of malodour production, a complete head and neck examination including nasal endoscopy, flexible laryngoscopyand site directed cultures are indicated as well as measurement and quantification of malodour. [2]

\section{-Biofilm and scraped specimens}

Bacteriological analysis from the biofilm and scrapped specimens obtained from the tongue dorsum or other oral sites can identify the VSC-producing bacteria. Prophyromonas, Prevotella, Actinobacillus and Fusobacterium species were the most common organisms identified from cultures. These were primarily on the tongue dorsum, gingival pockets and tonsil crypts. . [2]

-Culture

The culture from the tongue is different than the culture from the periodontal plaques. Treponema denticola, Prophyromonas gingivalis and Bacteriodes for causing the bacteria are found on the teeth, whereas anaerobic as Prevotella are found on tongue. [2]

-PCR (Polymerase Chain Reaction)

It is the most widely used technique in molecular biology. It is rapid, inexpensive, simple and canproduce relatively large numbers of couples of DNA molecules even when the source of DNA is relatively poor quality (eg: saliva or tongue coating).PCR provides quantitative analysis of 5 common bacteria responsible 
for oral malodour in saliva and tongue coat. These are as follows: P.gingivalis, Tanarella forsythia, Fusobacteriumnucleatum, Prevotellaintermedia and T.denticola. The results suggest that this array system for analysing the relationship between oral bacteria and halitosis is a helpful tool for monitoring the effectiveness of different therapeutic modalities. [2]

\section{Prevention:}

One of the common methods to prevent bad breath isBrush and floss more frequently,scrap the tongue,avoid foods that sour the breath,kick the habit of smoking,rinse the mouth using antibacterial mouthwash andskip after dinner mints and chew gums instead.[3]

\section{Treatment:}

1.Good Oral Hygiene Practices

Tooth Brush

Patients have to brush teeth at least twice a day using a fluoride-toothpaste.The brush has to be small to medium sized. It should have soft, multi tufted nylon bristles. The toothbrush has to be replaced periodically.A tooth brushing session has to last for at least two minutes. Makesure all areas are covered, especially where the teeth meets gums. Use a back and forth brushing motion on the outside and inside of the teeth. Lightly brush the tongue with a tongue scrapper or a separate toothbrush. ${ }^{[7]}$

\section{Floss}

Floss teeth once a day with dental floss to remove any trapped food. Flossing will reach areas brushing can't, such as along the gum line between teeth. Get a strand about 18inches long and wrap the ends of the strand around the middle finger of each hand until there is about 1inch of floss between each hand. Gently push the floss in between teeth, 'sawing' back and forth until the gum line is reached. When the floss reaches there, scrape the side of each teeth in a up-down motion. Keep moving to new sections of the strand so that not the same part of the floss is used. ${ }^{[7]}$ In one study, subjects who flossed were found to have significantly less mouth odor $(\mathrm{P}=0.016)$, saliva odor $(\mathrm{P}<0.001)$ and salivary cadaverine levels $(\mathrm{P}=0.011)$ than those who did not. ${ }^{[2]}$

Denture

Take dentures out at night so that mouth can recover.Do not clean dentures with toothpaste as it will scratch and stain them.Clean dentures thoroughly with soap and warm water, a denture cream or a denturecleaning tablet. ${ }^{[7]}$

\section{Mechanical Reduction}

Mechanical reduction of malodor can be achieved by reducing the intraoral bacterial count bydecreasing the production of VSCs. The common method used includes tongue scraping, tongue brushing and chewing gums. The first two methods should be made a daily routine. [4]

\section{Mouthwash}

Mouthwash also can use an anti-bacterial mouthwash.Mouthwashes suggested for the treatment of oral malodor act by reducing either the bacterial load or the associated odoriferous compounds. Chlorohexadienegluconate produces a fall in bacteria that produce VSC. A mouthwash of Chlorohexadiene and zinc lactate also reduces the oral malodor.Patients be reluctant to use Chlorohexadiene long term as it has an unpleasant taste, can give rise to a burning sensation. The best time to use a mouthwash is probably before bedtime because the residue of the mouth rinse will remain in the mouth for a longer period of time and have a greater effect and because the bacterial activity leading to bad breath is greatly during bedtime, when saliva flow is the highest. [4]

\section{Active Chemical Agents}

- In addition to mechanical means and normal oral hygiene procedures, the reduction of oral malodor is achieved by the use of active chemical agents. They include the delivery of the active microbial compounds via mouth rinse, dentifrices or lozenges. These compounds decrease the bacterial load thus decrease the VSC and VOC production.[4]

5. Diet

Other methods to reduce halitosis include changes in diet (reducing substrate for VOC production ) especially in a high-protein diet. Preventing the drying of oral mucous by hydration and sti8mulation of salivary flow can improve oral malodor due to presence of lysosomes in saliva, causing inhibition of bacterial growth. [4] 
Treatment Needs For Breath Malodor

\begin{tabular}{|l|l|}
\hline Category & Description \\
\hline TN-1 & $\begin{array}{l}\text { Explanation of halitosis and instructions for oral hygiene( support and reinforcement of all patient's } \\
\text { own self-care for further improvement of their oral hygiene) }\end{array}$ \\
\hline TN-2 & $\begin{array}{l}\text { Oral Prophylaxis , professional cleaning and treatment for oral diseases, especially periodontal } \\
\text { diseases. }\end{array}$ \\
\hline TN-3 & Referral to a physician or medical specialist. \\
\hline TN-4 & Explanation of examination data, further professional instruction, education and reassurance. \\
\hline TN-5 & Referral to a clinical psychologist, psychiatrist or other psychological specialist. \\
\hline
\end{tabular}

Ken Yaegakietal 2000[8]

\section{Discussion}

Halitosis can be an important social problem in which the standard dental treatments and mouthwashes that are often recommended provide only temporary relief. Oral malodor is primarily the result of microbial metabolism. Mouth is a home to hundreds of bacterial species with various nutritional preferences. These organisms digest proteins leading to bad breath. Oral malodor from the over growth of proteolytic, anaerobic gram negative bacteria onthe crevices of the tongue dorsum can be successfully diagnosed and treated.

Studies of the treatment for oral malodor have tended to report only short term data (weeks to a few months). Clearly, however, patients have to continue to maintain an effective oral hygiene regimen.

Bad breath is usually more of a nuisance rather than a serious medical condition. Most individuals with bad breath can treat and eliminate the condition on their own. Additionally, dentists and physicians can help with cases of chronic bad breath.

Experts say that at least half of us have halitosis at some point of our lives. Meals with strong tasting food, such as onions and garlic may result in what many people may consider as bad breath. Lifestyle may also influence whether certain kinds of breaths are smelly and unpleasant: a non-smoker finds a smoker's breathto be foul smelling while a smoker may not. However smoking is linked to a higher risk of dry mouth, dental and gum diseases which can cause bad breath. [2,4]

\section{Conclusion:}

In a nutshell, bad breath is an unpleasant odor which is source of microbial putrefaction of food debris, dells, saliva, blood and role of volatile sulfur compounds (VSCs) producing bacteria. Some common symptoms of bad breath includes bad breath smell, bad taste, or taste change, dry mouth and a coating on the tongue. Bad breath caused by diet, decrease flow of saliva, smoking, dry mouth, dentures or oral appliances and lastly, morning breath. Bad breath can be prevented by maintaining good oral hygiene, quit smoking, keep mouth moist and avoid food such as onions and garlic. Halitosis can be successfully diagnosed and treated.

\section{References}

[1]. Halitosis article | Bad breath \&Halitosis|Colgate |Oral Care Information.American Dental Association,2 002 - 2013 Aetna,Inc

[2]. AuthorAshutoshkackerMD;ChiefEditor:Arlen D Meyers,Halitosis,MedScape,Updated Nov 8,2012

[3]. PeteJanet,Improve Oral Health \&What You Can Do About Bad Brath,WebMD Feature, 2005 - 2013 WebMd

[4]. S R Porter and C Scully, Oral Malodour(Halitosis),2006 September 23

[5]. JohnP.Cunha,The American Dental Association "Bad Breath (halitosis)",MedicineNet.com

[6]. BreathOdour|University of Maryland Medical center

[7]. Christian Nordqvist,Medical News Today, 7 Oct 2009

[8]. Ken Yaegaki DDS,PHD and JefreyM.Coil,Examination Classification and Treatment of Halitosis;Clinical Perspectives ,J Can Dental Association, 2000. 